\title{
Stochastic homogenization of the laser intensity to improve the irradiation uniformity of capsules directly driven by thousands laser beams
}

\author{
M. Temporal ${ }^{1, a}$ and B. Canaud ${ }^{2}$ \\ 1 Universidad Politécnica de Madrid, 28040 Madrid, Spain \\ ${ }^{2}$ CEA, DAM, DIF, 91297, Arpajon, France
}

\begin{abstract}
Illumination uniformity of a spherical capsule directly driven by laser beams has been assessed numerically. Laser facilities characterized by $N_{D}=12,20,24,32,48$ and 60 directions of irradiation with associated a single laser beam or a bundle of $N_{B}$ laser beams have been considered. The laser beam intensity profile is assumed super-Gaussian and the calculations take into account beam imperfections as power imbalance and pointing errors. The optimum laser intensity profile, which minimizes the root-meansquare deviation of the capsule illumination, depends on the values of the beam imperfections. Assuming that the $N_{B}$ beams are statistically independents is found that they provide a stochastic homogenization of the laser intensity associated to the whole bundle, reducing the errors associated to the whole bundle by the factor $1 / N_{B}^{1 / 2}$, which in turn improves the illumination uniformity of the capsule. Moreover, it is found that the uniformity of the irradiation is almost the same for all facilities and only depends on the total number of laser beams $N_{\text {tot }}=N_{D} \times N_{B}$.
\end{abstract}

\section{Introduction}

In the last few decades a great effort has been dedicated to look for new and efficient ways to compress and ignite a small amount of thermonuclear deuterium-tritium (DT) fusion fuel in the context of the inertial confinement fusion (ICF) [1-5]. As it is well known, their aim is to provide the ignition and consequent burn wave propagation through a small mass of DT contained inside a spherical capsule. The final goal is to achieve high energy gain $(G=100)$. Two schemes have been originally proposed: the direct drive (DD) and the indirect drive (ID). In the DD scheme laser beams irradiate directly the capsule while in the ID scheme a fraction of the laser beam energy is first converted in X-rays which then irradiate the capsule. In the central ignition scheme a small amount of fuel (hot-spot) should reach the ignition condition provided that the capsule compression remains sufficiently stable during the implosion process. The direct drive scheme is more efficient than the indirect drive but it is also less robust with respect to hydrodynamic instabilities, such as Richtmyer-Meshkov [6,7] and Rayleigh-Taylor [8,9], that make more difficult the formation of an efficient hot-spot. These instabilities are initialized by both, irradiation nonuniformity as well as capsule imperfections. Recently, a different scheme known as shock ignition (SI) [10] has been proposed. In this new scheme the capsule is directly driven by two laser pulses with different tasks. A first laser pulse assembles the fuel but without thermonuclear ignition. After the fuel compression, a second high-power laser beam drives a strong shock devoted to ignite the pre-compressed DT fuel. One of the advantages of the Shock Ignition is that the self-ignition is not necessary and the requirements in term of capsule and irradiation uniformity are relaxed [11].

The use of the fusion energy provided by an ICF-based reactor imposes to work with a high-repetition rate facility. Indeed, the power $\left(P_{W}\right)$ supplied by a reactor should be proportional to the product of three factors, $E_{L} \times G \times F$, where $E_{L}$ is the laser energy, $G$ is the gain and $F$ is the laser repetition rate. Lets assume $P_{W}=1 \mathrm{GW}$, $E_{L}=1 \mathrm{MJ}, G=100$ and that $30 \%$ of the fusion energy will contribute to the net power plant, it turns out that the repetition rate $F$ will be of few tens Hz. High laser repetition rates affect the architecture of a future laser facility and impose the use of relatively small beamlets (laser beam units). Assuming relatively small laser beams, with surfaces of about $10^{2}-15^{2} \mathrm{~cm}^{2}$ and maximum energy fluence of $1 \div 3 \mathrm{~J} / \mathrm{cm}^{2}$, it turns out that a total of a few thousand beamlets should be used to provide a total energy of about 1 MJ. These beamlets should be grouped in bundles which surface would be of about $1-1.5 \mathrm{~m}^{2}$ (which is roughly the double of a quad in the National Ignition Facility [12] in the USA or the Laser MegaJoule [13] in 

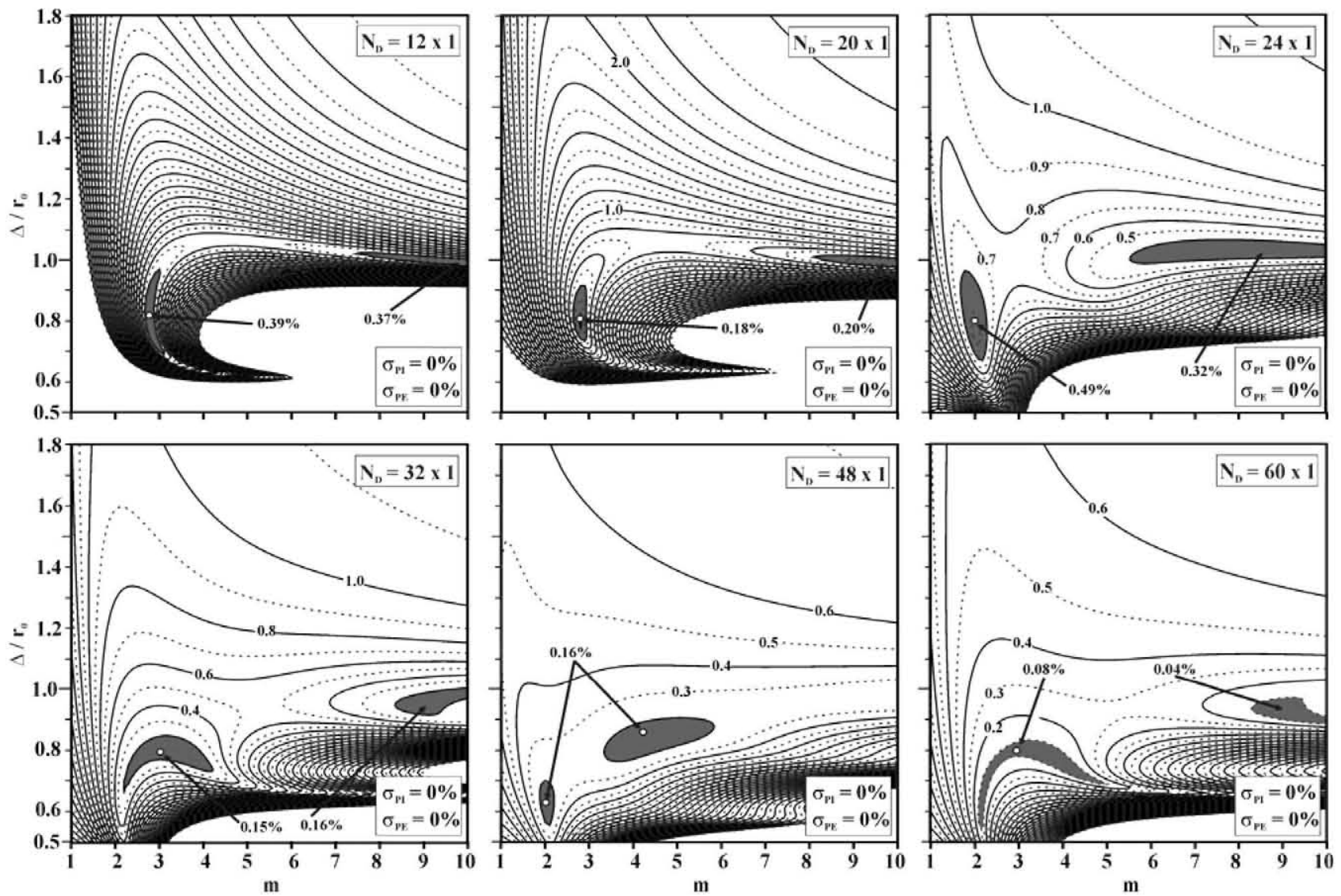

Fig. 1. (Color online) Intrinsic non-uniformity $\sigma_{0}$ evaluated for the configurations characterized by $N_{D}=12,20,24,32,48$ and 60 irradiation directions. The green areas are located at the minima of $\sigma_{0}$.

France). Moreover, the large number of beamlets is also useful in order to use the zooming technique [14] where different focal spots are used to optimize the laser-capsule coupling preserving the irradiation uniformity of the capsule [15].

In this paper we analyze the illumination nonuniformity of a spherical capsule directly irradiated by a large number of laser beams affected by unavoidable beam imperfections. Provided that these beams are organized in bundles, they generate a stochastic homogenization of the bundle laser intensities which reduces the irradiation nonuniformity.

\section{Illumination non-uniformity}

The uniformity of the illumination provided by six laser facilities has been numerically evaluated. In this case we neglect any effect originated by expansion of the plasma, thus these results are relevant for the imprinting phase of the irradiation which holds during the first few ns of the laser pre-pulse. The facilities, whose beam geometries are detailed in reference [16], are characterized by $N_{D}=12$ (based on the dodecahedron and used in the Gekko XII facility), $N_{D}=20$ (icosahedron), $N_{D}=24$ (omega facility), $N_{D}=32$ (dodecahedron + icosahedron), $N_{D}=48$ and
$N_{D}=60$ directions of irradiation (omega Upgrade). The illumination uniformity provided by the six facilities has been numerically calculated as the root mean square (rms) deviation $\sigma=\left[\int(I-\underline{I})^{2} d \Omega\right]^{1 / 2} /\left[(4 \pi)^{1 / 2} \underline{I}\right]$ of the laser intensity over a sphere of radius $r_{0}(\underline{I}$ is the average intensity and the integral covers the whole sphere surface) [17-23]. The intrinsic non-uniformity $\sigma_{0}$, which is a characteristic of each facility, has been calculated neglecting any laser imperfection as e.g. beam-to-beam power imbalance $\left(\sigma_{P I}\right)$ and pointing error $\left(\sigma_{P E}\right)$. For a given facility, the intrinsic non-uniformity depends only on the laser beam intensity profile, and represents the minimum non-uniformity theoretically achievable. Indeed, it corresponds to ideal facilities with perfectly balanced $\left(\sigma_{P I}=0\right)$ and centered laser beams $\left(\sigma_{P E}=0\right)$.

In our calculations we assumed a super-Gaussian laser intensity profile $I(r)=I_{0} \exp -(r / \Delta)^{m}$, characterized by the half width at $1 / e(\Delta)$ and by the exponent $m$. The intrinsic non-uniformity has been calculated as a function of the laser parameters $\left[\Delta / r_{0}, m\right]$, where $r_{0}$ is the capsule radius, and the parametric space has been limited to $1 \leqslant m \leqslant 10$ and $0.5 \leqslant \Delta / r_{0} \leqslant 1.8$. In Figure 1 the contour plots of the intrinsic non-uniformities $\sigma_{0}$ are shown for each configuration. In six frames the contour lines are separated by $0.1 \%$ and only contour lines with non-uniformities up to $\sigma_{0}=5 \%$ are shown. For each 

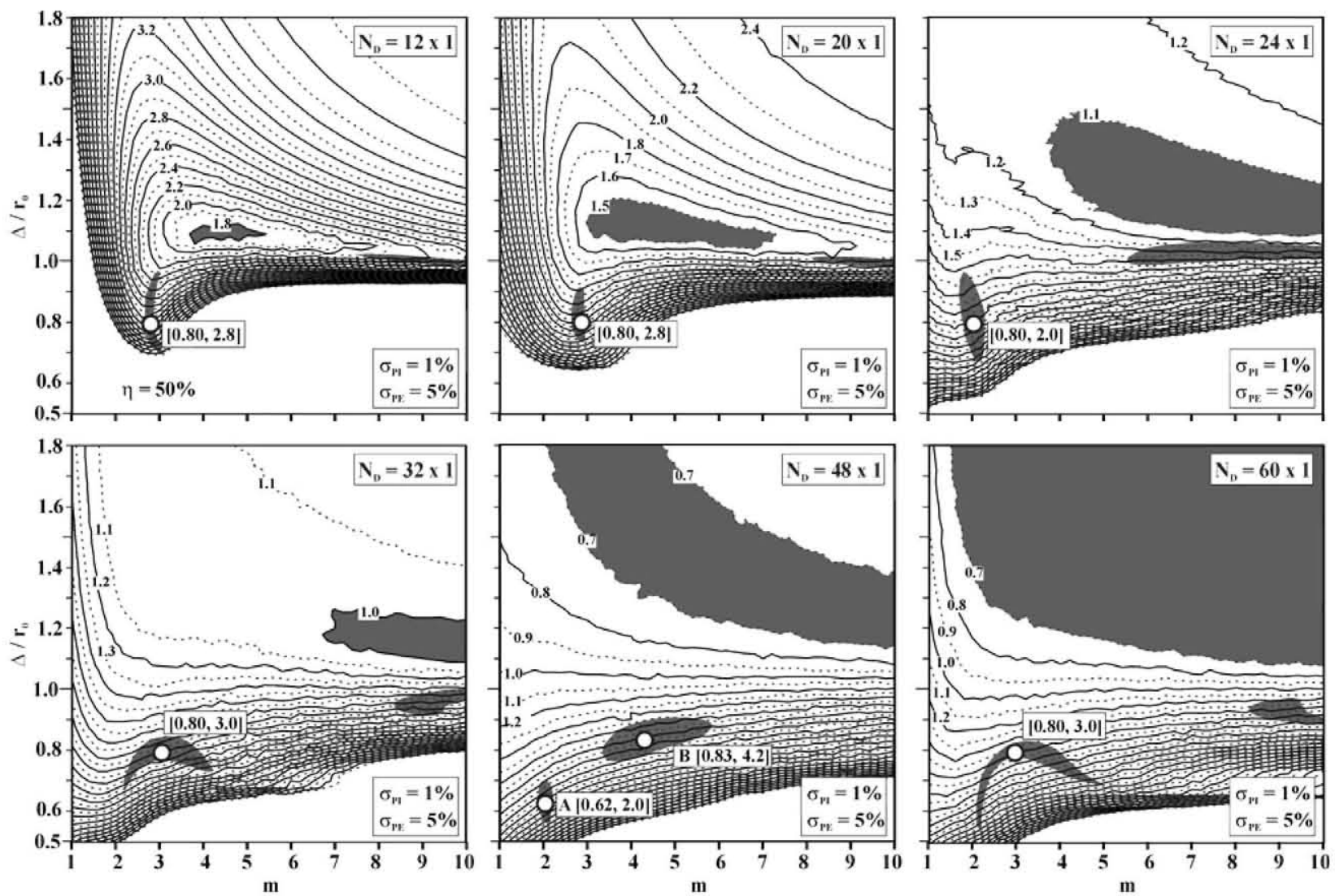

Fig. 2. (Color online) Non-uniformity $\sigma_{1}$ accounting for the beams imperfections. The green areas show the position of the minima of the intrinsic non-uniformity $\sigma_{1}$.

laser facility the intrinsic non-uniformity exhibits two minima in the parametric space $\left[\Delta / r_{0}, m\right]$. The smaller values of the intrinsic non-uniformity have been found for the configurations with $N_{D}=20,32,48$ and 60 where $\sigma_{0}<0.2 \%$, while higher values are found for the $N_{D}=12$ and $N_{D}=24$. The positions of these minima are indicated by the green areas in the frames of Figure 1 .

These calculations refer only to ideal configurations assuming that all laser beams are equal and perfectly centered, thus neglecting any beam imperfections. In any real configuration such errors cannot vanish and hereafter, we will consider $\sigma_{P I}=1 \%$ and $\sigma_{P E}=5 \%$ as reasonable values for the power imbalance and the pointing error. Therefore, if the capsule radius is $1000 \mu \mathrm{m}$, the expected root-mean-square deviation of the pointing error is about $50 \mu \mathrm{m}$. Calculations have been performed to evaluate the degradation of the illumination uniformity when the power imbalance $\sigma_{P I}=1 \%$ and the pointing error $\sigma_{P E}=5 \%$ are associated to $N_{D}$ laser beams. For each facility, a set of thousand calculations have been performed assuming that the laser power and the pointing errors follow a random Gaussian distribution characterized by the standard deviations $\sigma_{P I}$ and $\sigma_{P E}$ respectively. Assuming a total of $N_{D}$ laser beams (one beam for irradiation directions, $N_{B}=1$ ) these calculations provide an average rms non-uniformities $\sigma_{1}$. Figure 2 shows the non-uniformity $\sigma_{1}$ as a function of the beam parameters. The green areas indicate the focal parameters that minimize the intrinsic non-uniformity $\sigma_{0}$ (same areas as in Fig. 1) which do not coincide with these that minimize the non-uniformity $\sigma_{1}$ (red areas).

The data associated to the beam intensity profile $\Delta / r_{0}$ and the exponent $m$ corresponding to the minimum of the intrinsic non-uniformity $\sigma_{0}$ have been summarized in Table 1. For each laser facility $\left(N_{D}\right)$ the minimum intrinsic non-uniformity $\sigma_{0}$ and the non-uniformities $\sigma_{1}$ accounting for the beam imperfections are shown. The nonuniformity $\sigma_{1}$ is larger than $1 \%$ for all the configurations. However, these non-uniformities $\left(\sigma_{1}\right)$ have been calculated for the optimum laser beam parameters that minimize $\sigma_{0}$ which in general do not correspond to the minimum of $\sigma_{1}$. The optimum laser intensity profile which minimizes $\sigma_{1}$ depends on the beam imperfections [23]. Indeed, for the beam imperfections $\sigma_{P I}=1 \%$ and $\sigma_{P E}=5 \%$ it is found that the minima of $\sigma_{1}\left[N_{D}\right]$ are: $\sigma_{1}[12] \approx 1.7 \%$ at $\left[\Delta / r_{0} \approx 1.2, m \approx 4\right] ; \sigma_{1}[20] \approx 1.4 \%$ at $\left[\Delta / \mathrm{r}_{0} \approx 1.1\right.$, $m \approx 4] ; \sigma_{1}[24] \approx 1.2 \%$ at $\Delta / r_{0}>1.3 ; \sigma_{1}[32] \approx 1.0 \%$ at $\left[\Delta / r_{0} \approx 1.5, m \approx 3\right] ; \sigma_{1}[48] \approx 0.7 \%$ for $\Delta / r_{0}>1.5 ; \sigma_{1}[60]$ $\approx 0.6 \%$ for $\Delta / r_{0}>1.5$. Thus, only the configurations with $N_{D}=48$ and $N_{D}=60$ exhibit non-uniformities smaller than $1 \%$ (but to the price of unreasonably large focal spots $\left.\Delta / r_{0}>1.5\right)$. The optimum laser parameters that minimize $\sigma_{1}$ change when the beam imperfections are modified. For all cases, the optimum laser beam parameters 


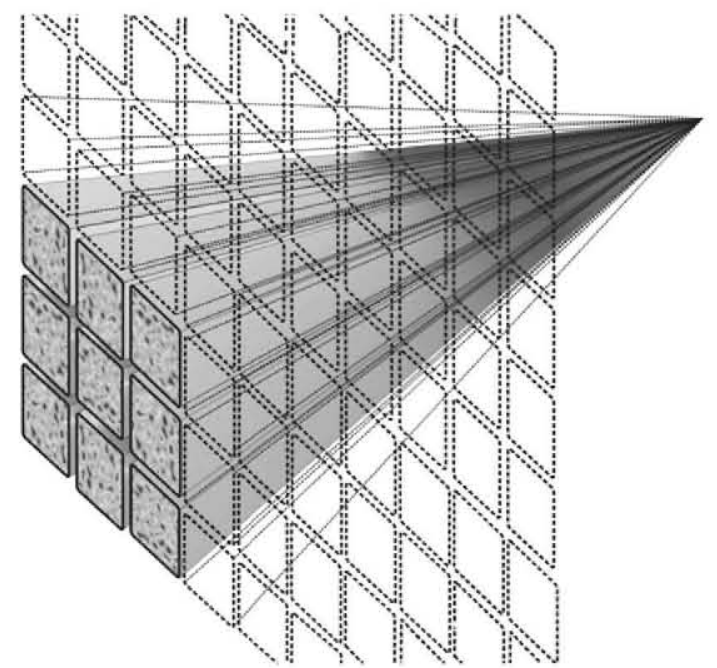

Fig. 3. (Color online) Sketch of a bundle with $10 \times 10$ beamlets.

Table 1. Optimum beam parameters for the minimum intrinsic non uniformity $\sigma_{0}$.

\begin{tabular}{ccccc}
\hline$N_{D}$ & $\Delta / r_{0}$ & $m$ & $\sigma_{0}$ & $\sigma_{1}$ \\
\hline 12 & 0.80 & 2.8 & 0.39 & 3.45 \\
20 & 0.80 & 2.8 & 0.18 & 2.67 \\
24 & 0.80 & 2.0 & 0.49 & 2.21 \\
32 & 0.80 & 3.0 & 0.15 & 2.12 \\
$48(\mathrm{~A})$ & 0.62 & 2.0 & 0.16 & 2.47 \\
$48(\mathrm{~B})$ & 0.83 & 4.2 & 0.16 & 1.85 \\
60 & 0.80 & 3.0 & 0.08 & 1.61 \\
\hline
\end{tabular}

move from large focal spots $\left(\Delta / r_{0}>1.1\right.$ at $\sigma_{P I}=1 \%$ and $\left.\sigma_{P E}=5 \%\right)$ towards the position of the minimum intrinsic non-uniformity as the errors tend to zero.

\section{Bundles}

In order to decrease the non-uniformity it is necessary to reduce the beam imperfections. An attractive natural way to reduce these errors consists in grouping $N_{B}$ laser beams in bundles associated to each direction or irradiation $N_{D}$ [24] so that the total number of beams is $N_{\text {tot }}=N_{D} \times N_{B}$. In Figure 3 it is shown a sketch of a bundle with $10 \times 10$ beamlets. In this work we assume that $N_{B}$ beamlets in a bundle are statistically independent. In the hypothesis that a power imbalance $\sigma_{P I}^{0}$ and a pointing error $\sigma_{P E}^{0}$ are assigned to each beamlet, it follows that the errors associated to each whole bundle are reduced by the statistic factor to $\sigma_{P I}=\sigma_{P I}^{0} / N_{B}^{1 / 2}$ and $\sigma_{P E}=\sigma_{P E}^{0} / N_{B}^{1 / 2}$. In this way a relatively large number of beamlets $N_{\text {tot }}=N_{D} \times N_{B}$ should be involved in the facility. As a consequence, a laser facility characterized by a single beam per direction of irradiation $\left(N_{B}=1\right)$ with given beam imperfections $\sigma_{P I}^{0}$ and $\sigma_{P E}^{0}$ provides the same illumination uniformity as a second one with hundred $\left(N_{B}=100\right)$ beamlets per direction of irradiation and ten times bigger errors.

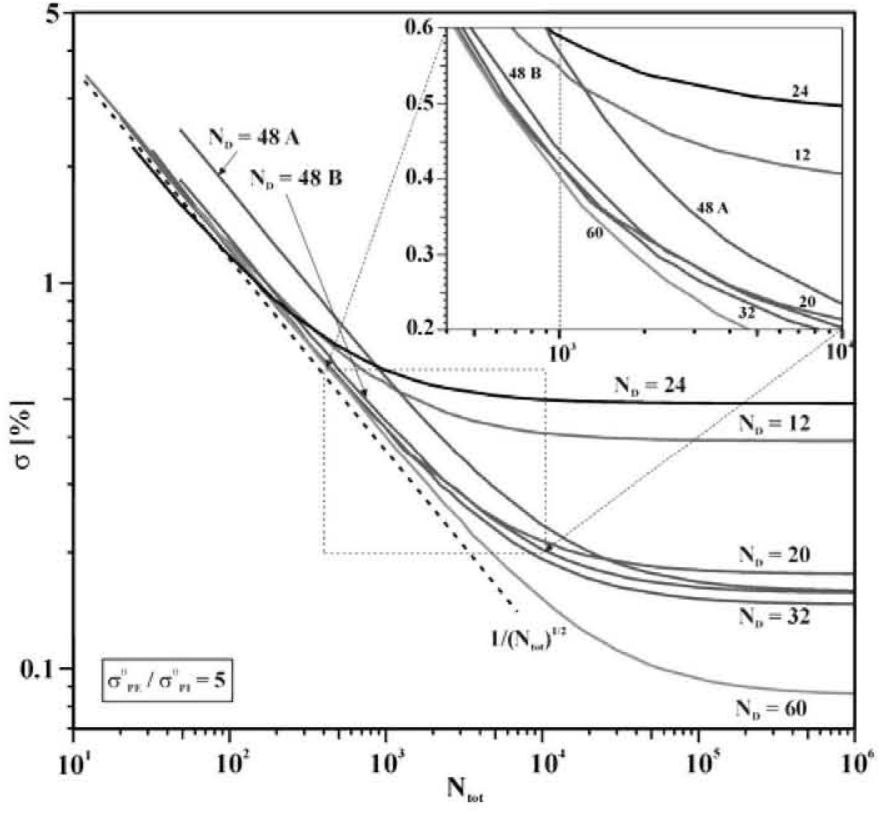

Fig. 4. (Color online) Uniformity $\left(\sigma_{1}\right)$ as a function of the total number of beamlets $\left(N_{\text {tot }}\right)$ all characterized by a power imbalance of $\sigma_{P I}^{0}=1 \%$ and pointing error $\sigma_{P E}^{0}=5 \%$.

A set of calculations has been performed assuming $\sigma_{P I}^{0}=1 \%$ and $\sigma_{P E}^{0}=5 \%$ and increasing the total number of beamlets from $N_{\text {tot }}=N_{D}$ to approximately $N_{\text {tot }}=10^{6}$. The super-Gaussian beam intensity profiles given in Table 1 and characterized by the parameters $\Delta / r_{0}$ and $m$ have been used in these calculations. They almost correspond to the optimum parameters for a total number of beams larger than one thousand. The calculations assume that each bundle acts as one laser beam with associated reduced errors given by: $\sigma_{P I}=\sigma_{P I}^{0} / N_{B}^{1 / 2}$ and $\sigma_{P E}=\sigma_{P E}^{0} / N_{B}^{1 / 2}$, where $N_{B}=N_{t o t} / N_{D}$. The nonuniformities $\sigma$ are shown in Figure 4 as a function of the total number of beamlets $N_{\text {tot }}$. It is shown that until about a few thousands beamlets all laser configurations, except for $N_{D}=12$ and $N_{D}=24$, provide almost the same non-uniformity that scales approximately as $1 / N_{\text {tot }}^{1 / 2}$. Moreover, modifying the ratio of the beam imperfections $\sigma_{P E}^{0} / \sigma_{P I}^{0}$ by a factor $F_{\varepsilon}$ the curves in Figure 3 shift, parallel to the horizontal axis, by a factor $F_{\varepsilon}^{2}$. As an example, if the characteristic errors of the facilities are doubled (or divided by a factor of two) that is $F_{\varepsilon}=2, \sigma_{P I}^{0}=2 \%$ and $\sigma_{P E}^{0}=10 \%$, the number of beamlets should be multiplied by $F_{\varepsilon}^{2}=4$ [or divided by four] in order to provide the same non-uniformities.

The three configurations with $N_{D}=12,24$ and $48 \mathrm{~A}$ provide the worse results. The first two are penalized by a too high intrinsic uniformity $\sigma_{0}$ while the $48 \mathrm{~A}$ is too instable with respect to the beam imperfections providing a large $\sigma_{1}$. For a large number of beamlets, roughly from $N_{\text {tot }}=500$ to $N_{\text {tot }}=3000$, the configurations with $N_{D}=20,32,48$ and 60 provide non-uniformities of $0.3-0.6 \%$. At about $N_{\text {tot }}=1000$ the best uniformities with $\sigma \approx 0.4 \%$ are attained by the configuration with $N_{D}=60$, followed by $N_{D}=32, N_{D}=20$ and then by the 
$N_{D}=48$. These non-uniformities are very well fitted by equation (1), which is the extension of the one developed in references $[20,21]$. In this equation, the non-uniformity is a combination of the intrinsic non-uniformity $\sigma_{0}^{2}$ plus a second term which accounts for the beam imperfections and scales as $1 / \mathrm{N}_{\text {tot }}^{1 / 2}$. Thus, increasing the number $N_{D}$ of irradiation directions decreases the first term $\left(\sigma_{0}^{2}\right)$, while increasing the number of beamlets $N_{B}$ in the bundles decreases the second term. The data (see Tab. 1) show that the quantity $\Delta \sigma$ is almost constant and equal to $12 \%$ for all configurations. With this value $(\Delta \sigma=12 \%)$ the equation reproduces very well the numerical data.

$$
\sigma=\sqrt{\sigma_{0}^{2}+\frac{(\Delta \sigma)^{2}}{N_{\text {tot }}}} ; \text { with } \Delta \sigma=\sqrt{N_{D}\left(\sigma_{1}^{2}-\sigma_{0}^{2}\right)} .
$$

\section{Conclusions}

The illumination uniformity of a spherical capsule directly driven by laser beams with a power imbalance of $\sigma_{P I}=1 \%$ and a pointing error of $\sigma_{P E}=5 \%$ has been analyzed. The calculations show that the optimum laser intensity profile depends on the beam imperfections. Laser facilities characterized by $N_{D}$ irradiation directions with associated bundles of $N_{B}$ beamlets have been considered. The use of a large number of beamlets $\left(N_{\text {tot }}=N_{D} \times N_{B}\right)$ is consistent with the constraints of a high-repetition rate facility. The basic assumption of this work is that each bundle is assembled from $N_{B}$ statistically independent beamlets affected by imperfections and that the final errors associated to the whole bundle are reduced by the statistic factor $1 / N_{B}^{1 / 2}$. For the laser intensity profile that optimizes the intrinsic illumination non-uniformity it is found that assuming only one beam per direction of irradiation (without bundles, $\left.N_{B}=1\right)$ the non-uniformity $\left(\sigma_{1}\right)$ is always bigger than $1 \%$ and increases when $N_{D}$ is reduced. Differently, the non-uniformity decreases when bundles with approximately a total of hundred to a few thousand beamlets are considered. For a very large number of total beams $\left(N_{\text {tot }} \geqslant 10^{4}\right)$ the uniformity is dominated by the intrinsic non-uniformity $\left(\sigma_{0}\right)$ which in general decreases with the number of directions of irradiation $N_{D}$. Finally, the use of a large number of beamlets $\left(N_{t o t}\right)$ provides a stochastic homogenization of the laser intensity of the whole bundle which in turn improves the uniformity of the capsule illumination. Thus, for a given total number of beamlets, the configurations characterized by $N_{D}=20,32,48$ and 60 directions of irradiations provide almost the same nonuniformity. Namely, they provide a uniformity of about $0.4 \%$ using a total number of laser beams of about one thousand.
This work has been performed in the framework of the HiPER project. M.T. has been supported by the research contracts "Ramon y Cajal" 2007-00447 and ENE2009-11668 of the Spanish Ministry of Education and Research (MEC).

\section{References}

1. J.H. Nuckolls, L. Wood, A. Thiessen, G.B. Zimmermann, Nature 239, 129 (1972)

2. J.D. Lindl, P. Amendt, R.L. Berger, S.G. Glendinning, S.H. Glenzer, S.W. Haan, R.L. Kauffman, O.L. Landen, L.J. Suter, Phys. Plasmas 11, 339 (2004)

3. J.D. Lindl, Inertial Confinement Fusion: The Quest for Ignition and high Gain Using Indirect Drive (Springer, New York, USA, 1998)

4. J. Lindl, Phys. Plasmas 2, 3933 (1995)

5. S. Atzeni, J. Meyer-ter-Vehn, The Physics of Inertial Fusion (Oxford University Press, Oxford, 2004)

6. R.D. Richtmyer, Commun. Pure Appl. Math. 13, 297 (1960)

7. E.E. Meshkov, Fluid Dyn. 4, 101 (1969)

8. Lord Rayleigh, Scientific Papers (Dover, New York, 1965)

9. G.I. Taylor, Proc. Roy. Soc. Lond. A 201, 192 (1950)

10. R. Betti, C.D. Zhou, K.S. Anderson, L.J. Perkins, W. Theobald, A.A. Solodov, Phys. Rev. Lett. 98, 155001 (2007)

11. B. Canaud, M. Temporal, New J. Phys. 12, 043037 (2010)

12. E.I. Moses, Energy Convers. Manag. 49, 1795 (2008)

13. J. Giorla, J. Bastian, C. Bayer, B. Canaud, M. Casanova, F. Chaland, C. Cherfils, C. Clique, E. Dattolo, P. Fremerye, D. Galmiche, F. Garaude, P. Gauthier, S. Laffite, N. Lecler, S. Liberatore, P. Loiseau, G. Malinie, L. Masse, A. Masson, M.C. Monteil, F. Poggi, R. Quach, F. Renaud, Y. Saillard, P. Seytor, M. Vandenboomgaerde, J. Van der Vliet, F. Wagon, Plasma. Phys. Control. Fusion 48, B75 (2006)

14. B. Canaud, F. Garaude, Nucl. Fusion 45, 43 (2005)

15. M. Temporal, B. Canaud, B.J. Le Garrec, Phys. Plasmas 17, $022701(2010)$

16. J. Xiao, B. Lu, J. Opt. 29, 282 (1998)

17. S. Skupsky, K. Lee, J. Appl. Phys. 54, 3662 (1983)

18. A.J. Schmitt, Appl. Phys. Lett. 44, 399 (1984)

19. M. Murakami, Appl. Phys. Lett. 66, 1587 (1995)

20. M. Murakami, K. Nishihara, H. Azechi, J. Appl. Phys. 74, $802(1993)$

21. B. Canaud, X. Fortin, N. Dague, J. Bocher, Phys. Plasmas. 9, $4252(2002)$

22. M. Temporal, B. Canaud, Eur. Phys. J. D 55, 139 (2009)

23. M. Temporal, B. Canaud, S. Laffite, B. Le Garrec, M. Murakami, Phys. Plasmas 17, 064504 (2010)

24. B.J. Le Garrec, C. Hernandez-Gomez, T. Winstone, J. Collier, J. Phys.: Conf. Ser. 244, 032020 (2010) 